\title{
Simulations of the infrared, Raman, and 2D-IR photon echo spectra of water in nanoscale silica pores
}

Paul C. Burris, Damien Laage, and Ward H. Thompson

Citation: The Journal of Chemical Physics 144, 194709 (2016);

View online: https://doi.org/10.1063/1.4949766

View Table of Contents: http://aip.scitation.org/toc/jcp/144/19

Published by the American Institute of Physics

\section{Articles you may be interested in}

IR and Raman spectra of liquid water: Theory and interpretation

The Journal of Chemical Physics 128, 224511 (2008); 10.1063/1.2925258

Solute location in a nanoconfined liquid depends on charge distribution

The Journal of Chemical Physics 143, 044701 (2015); 10.1063/1.4926936

Water-anion hydrogen bonding dynamics: Ultrafast IR experiments and simulations

The Journal of Chemical Physics 146, 234501 (2017); 10.1063/1.4984766

Simulating two-dimensional infrared-Raman and Raman spectroscopies for intermolecular and intramolecular modes of liquid water

The Journal of Chemical Physics 144, 074201 (2016); 10.1063/1.4941842

Removing the barrier to the calculation of activation energies

The Journal of Chemical Physics 145, 134107 (2016); 10.1063/1.4964284

Comparison of simple potential functions for simulating liquid water

The Journal of Chemical Physics 79, 926 (1998); 10.1063/1.445869

\section{AIP| The Joumal of Chemical Physics}

PERSPECTIVES 


\title{
Simulations of the infrared, Raman, and 2D-IR photon echo spectra of water in nanoscale silica pores
}

\author{
Paul C. Burris, ${ }^{1}$ Damien Laage, ${ }^{2, a)}$ and Ward H. Thompson ${ }^{1, b)}$ \\ ${ }^{1}$ Department of Chemistry, University of Kansas, Lawrence, Kansas 66045, USA \\ ${ }^{2}$ Département de Chimie, Ecole Normale Supérieure-PSL Research University, Sorbonne Universités-UPMC \\ Univ Paris 06, CNRS UMR 8640 PASTEUR, 24 rue Lhomond, 75005 Paris, France
}

(Received 1 March 2016; accepted 3 May 2016; published online 20 May 2016)

\begin{abstract}
Vibrational spectroscopy is frequently used to characterize nanoconfined liquids and probe the effect of the confining framework on the liquid structure and dynamics relative to the corresponding bulk fluid. However, it is still unclear what molecular-level information can be obtained from such measurements. In this paper, we address this question by using molecular dynamics (MD) simulations to reproduce the linear infrared (IR), Raman, and two-dimensional IR (2D-IR) photon echo spectra for water confined within hydrophilic (hydroxyl-terminated) silica mesopores. To simplify the spectra the $\mathrm{OH}$ stretching region of isotopically dilute $\mathrm{HOD}$ in $\mathrm{D}_{2} \mathrm{O}$ is considered. An empirical mapping approach is used to obtain the $\mathrm{OH}$ vibrational frequencies, transition dipoles, and transition polarizabilities from the MD simulations. The simulated linear IR and Raman spectra are in good general agreement with measured spectra of water in mesoporous silica reported in the literature. The key effect of confinement on the water spectrum is a vibrational blueshift for $\mathrm{OH}$ groups that are closest to the pore interface. The blueshift can be attributed to the weaker hydrogen bonds (H-bonds) formed between the $\mathrm{OH}$ groups and silica oxygen acceptors. Non-Condon effects greatly diminish the contribution of these $\mathrm{OH}$ moieties to the linear IR spectrum, but these weaker $\mathrm{H}$-bonds are readily apparent in the Raman spectrum. The 2D-IR spectra have not yet been measured and thus the present results represent a prediction. The simulated spectra indicates that it should be possible to probe the slower spectral diffusion of confined water compared to the bulk liquid by analysis of the 2D-IR spectra. Published by AIP Publishing. [http://dx.doi.org/10.1063/1.4949766]
\end{abstract}

\section{INTRODUCTION}

Liquids confined on nanometer length scales exhibit differences with respect to the corresponding bulk liquid in nearly every structural and dynamical characteristic. ${ }^{1-8}$ This represents a tremendous opportunity for controlling the liquid properties through the confining framework attributes, but with the associated challenge of understanding the connection between the two. One of the key limiting factors is the availability of experimental measurements that can provide detailed, molecular-level understanding of nanoconfined liquid properties. Theoretical studies can be of great value in this context by connecting the results of measurements to molecular properties, a key step in interpreting experimental signals.

In this paper we focus on this problem in the context of vibrational spectroscopic measurements of water confined within mesoporous silica. Infrared and Raman spectroscopy are commonly used to probe the properties of confined liquids, ${ }^{9-29}$ yet the interpretation at a molecular scale is not always clear. ${ }^{30-32}$ This is a considerable, outstanding obstacle in characterizing nanoconfined liquid structure and dynamics.

Among the changes experienced by a liquid upon confinement is layering induced by the interface with the

\footnotetext{
a) Electronic mail: damien.laage@ens.fr

b) Electronic mail: wthompson@ku.edu
}

confining framework, which is also generally associated with orientational ordering. ${ }^{32-39}$ In addition, the reorientational and diffusional dynamics are slowed, often dramatically. ${ }^{10,39-49}$ The effect of nanoscale confinement on water is more limited to the interfacial region compared to other liquids despite the fact that it is a networked liquid. ${ }^{22,39,46,47}$ This can primarily be attributed to the absence in water of steric effects that propagate the constraints of the confining surface to larger distances in other liquids.

We have previously reported simulations of the linear infrared (IR) spectrum of the $\mathrm{CN}$-stretch of acetonitrile in the same hydrophilic silica pores as considered here ${ }^{32}$ that are in good agreement with experimental measurements. ${ }^{10,15}$ The key effect of confinement on the simulated spectrum compared to that of the bulk liquid is the appearance of a new peak at higher frequency for molecules accepting a hydrogen bond from a surface silanol. This frequency blue-shift is due to the special property of nitriles in hydrogen bonds, in which charge transfer from the nitrogen lone pair (which has some $\pi^{*}$ character) to the hydrogen-bond donor results in a stronger $\mathrm{CN}$ bond. Otherwise, the spectrum shows little effect of confinement on acetonitrile molecules that were not engaged in hydrogen bonding. It is interesting then to compare the case of acetonitrile with that of water, which exhibits different details in the structural and dynamics effects upon confinement as well as distinct vibrational spectroscopic properties. 
A number of simulations of water confined in silica have been previously reported, ${ }^{38,39,46,47,50-55}$ but the vibrational spectra has not been modeled. Here we present simulations of the linear IR and Raman spectra along with the twodimensional infrared (2D-IR) spectra in the $\mathrm{OH}$-stretching region of dilute $\mathrm{HOD}$ in $\mathrm{D}_{2} \mathrm{O}$ confined within $\sim 2.4 \mathrm{~nm}$ hydrophilic, amorphous silica pores. Both the linear IR and Raman spectra have been measured for $\mathrm{H}_{2} \mathrm{O}$ in mesoporous silica and provide an opportunity to validate the simulation approach. Experimental 2D-IR spectra have not yet been reported, so the simulated spectra here represent a prediction. The key aspect of the present work, however, is to examine what properties of the confined water are (and are not) reported on by these different vibrational spectroscopies. This should aid in both the design and interpretation of vibrational spectroscopy experiments to probe nanoconfined liquids.

\section{SIMULATION METHODOLOGY}

Classical molecular dynamics simulations were carried out using the LAMMPS code. ${ }^{56,57}$ Two water-filled, hydrophilic (OH-terminated) silica pores of diameter $\sim 2.4 \mathrm{~nm}$ were used for the simulations presented here; both were modeled in a rectangular periodic simulation box with dimensions of $30 \AA$ along the pore axis and $44 \AA$ in the perpendicular directions. The two pores were created with the same procedure and differ only in the atomic-level structure. ${ }^{31}$ The number of water molecules was determined by grand canonical Monte Carlo simulations using the Towhee program ${ }^{58}$ with a chemical potential of $-30 \mathrm{~kJ} / \mathrm{mol}$, a value that was chosen because it gives a fully water-filled pore (441 molecules for the pore hereafter referred to as pore 1 and 414 for pore 2); changing the chemical potential around this value yields only minor differences in the number of water molecules in the pore. The structure of pore 1 is shown in Fig. 1 along different cross-sectional cuts.

The SPC/E model ${ }^{59}$ was used to describe the water interactions. The parameters for the silica force field have been given previously. ${ }^{31,60}$ Intermolecular interactions were evaluated with a cutoff of $12 \AA$ and long-range electrostatic interactions were included using three-dimensional periodic boundary conditions using an Ewald summation with a tolerance of $10^{-4}$.

Five $1 \mathrm{~ns}$ trajectories were propagated with a $1 \mathrm{fs}$ timestep for water confined in pore 1 and one $1 \mathrm{~ns}$ trajectory for water in pore 2 ; the trajectories were initiated from a previously reported $20 \mathrm{~ns}$ trajectory, ${ }^{47}$ with the trajectories varying in the length of two subsequent equilibration stages, the first of 0-2 ns with velocity rescaling and the second $0.5-5 \mathrm{~ns}$ in the NVT ensemble. The NVT ensemble used a Nosé-Hoover thermostat $^{61,62}$ with a time constant of 1 ps to maintain the temperature at $298.15 \mathrm{~K}$ before and during the data collection stage. The linear IR and Raman spectra (Secs. III B and III C) from the five $1 \mathrm{~ns}$ trajectories are nearly identical, indicating that a single trajectory is sufficient to calculate these results. The two-dimensional infrared (2D-IR) spectra (Sec. III D) from each trajectory are quantitatively similar, however, they benefit from the additional averaging, as do distance-dependent quantities.

Bulk water simulations were carried out to provide a comparison with the confined water results. Three $1 \mathrm{~ns}$ trajectories were run with 343 molecules in a cubic box of length $21.725 \AA$ with a 1 fs timestep in the NVT ensemble at $298.15 \mathrm{~K}$ as described above for the pore system. The trajectories differed in the length of an equilibration stage of 0.25-0.75 ns. The intermolecular interactions were evaluated with a cutoff of $10.5 \AA$ and long-range electrostatic interactions were included using three-dimensional periodic boundary conditions with an Ewald summation with a tolerance of $10^{-4}$.

\section{A. Frequency map and implementation}

To obtain the quantities required for calculating the linear and nonlinear vibrational spectra, we use the empirical mapping approach of Skinner and co-workers. ${ }^{63-65}$ The vibrational transition frequencies of an $\mathrm{OH}$ group are then related to the electric field imposed on the $\mathrm{H}$ atom by the surrounding waters and, in this work, the silica pore atoms. Specifically, we employ the quadratic map ${ }^{64,65}$ such that at a given time $t$ the fundamental vibrational frequency on the $i$ th $\mathrm{OH}$ is,

$$
\omega_{01}^{(i)}(t)=3761.6-5060.4 \mathcal{E}_{i}(t)-86225 \mathcal{E}_{i}(t)^{2},
$$

where $\omega_{01}$ is in $\mathrm{cm}^{-1}$ and the electric field,

$$
\mathcal{E}_{i}(t)=\overrightarrow{\mathbf{e}}_{O H, i}(t) \cdot \overrightarrow{\mathcal{E}}_{t o t}\left(\mathbf{r}_{H, i}, t\right)
$$

is input in atomic units. Here, $\mathcal{E}_{t o t}$ is the total electric field and $\mathbf{r}_{H, i}$, and $\mathbf{e}_{O H, i}$ are the location of the hydrogen atom and the unit vector (pointing towards $\mathrm{H}$ ) of the $i$ th $\mathrm{OH}$ group. Similar expressions to Eq. (1) are used to relate the projected
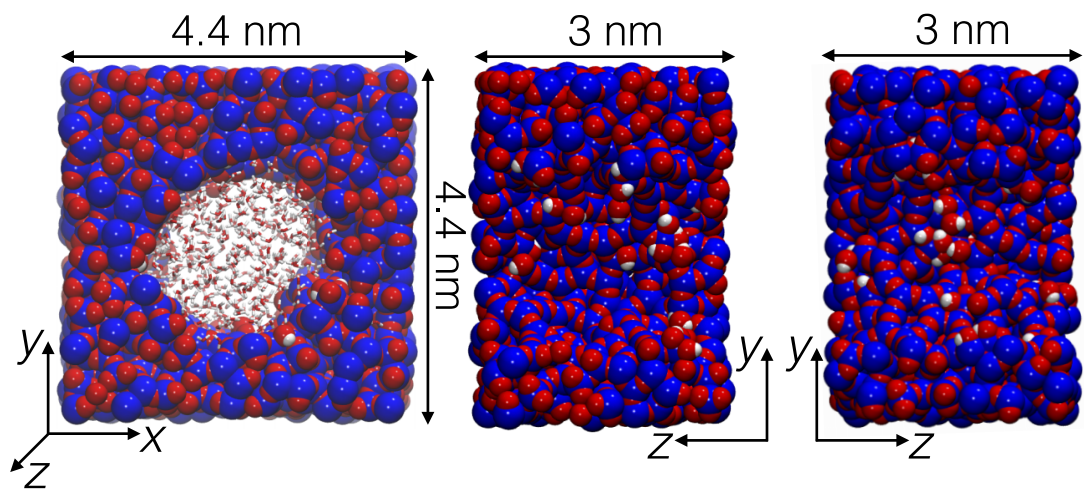

FIG. 1. The structure of pore 1 used in the present simulations is shown along three cross-sectional directions: looking down the water-filled pore $(z)$ axis (left), looking at the left $(x<0)$ crosssection along the pore axis (center), and looking at the right $(x>0)$ cross section along the pore axis (right). The coordinate axes are defined in each case, the simulation cell dimensions are indicated, and $\mathrm{Si}$ (blue), $\mathrm{O}$ (red), and $\mathrm{H}$ (white) atoms are shown. 
electric field $\mathcal{E}_{i}$ to $\omega_{12}$, the transition dipole moment, and the transition polarizability (parameters are given in Ref. 64).

We note that the direct contribution to the spectra from surface silanols are not considered in the calculations, e.g., the infrared absorption by the silanol $\mathrm{OH}$ groups is not included in the spectra reported here (and similarly for the Raman and 2D-IR spectra). Simple estimates suggest that they would at best have a small effect on the spectra that would be difficult to discern in a fully hydrated pore such as that considered here. This is supported by experimental measurements ${ }^{15,23,24}$ and the number densities (e.g., in the two pores considered here there are only 56 and 86 silanol groups compared to 882 and 818 water $\mathrm{OH}$ groups, respectively). However, the electric field exerted by the silanols on the $\mathrm{OH}$ groups is included in the determination of the water $\mathrm{OH}$ stretching frequencies, with the exception of the case described below where the field due to the entire silica pore is neglected.

The empirical maps were derived for neat water, yet we have used them without modification for just such interfacial waters as a first-order estimate of the spectroscopic properties of water near a silica surface. Future work will be devoted to the development of a frequency map specifically designed for water next to silica, but we will show below that the approximate description with the bulk frequency map already provides a faithful representation of the pore influence on the water vibrational spectra. It is useful, however, to consider the sensitivity of the implementation. In the case of neat water, the total electric field, $\mathcal{E}_{t o t}$, is calculated from all waters within a cut-off distance of $R_{\text {cut }}=7.831 \AA$ of the $\mathrm{H}$ atom of interest. ${ }^{64}$ We have used this approach, except where noted, for all the results in this paper where we include not only all waters, but all atoms making up the silica pore within this cut-off distance. In addition, we have also calculated the vibrational spectra by including the electric field from all silica pore atoms, while only water molecules within $R_{\text {cut }}$ are included. We have also used the approach implemented by Skinner and co-workers for water in salt solutions and near lipid headgroups in which the partial charges of the ions and lipid sites are scaled separately for positive (by 0.81379) and negative (by 0.92017) sites. ${ }^{66-68}$ Finally, for purposes of comparison, we have fully neglected the field from the silica pore atoms to yield only the frequency shifts generated by the water molecules.

The distribution of $\mathrm{OH}$ fundamental frequencies,

$$
P(\omega)=\left\langle\delta\left(\omega-\omega_{01}\right)\right\rangle,
$$

is displayed in Fig. 2 for each of these four methods of calculating the electric field. The bulk water frequency distribution is also plotted for comparison. (Analogous results are shown for the 2D-IR spectra in Fig. S1 of the supplementary material. ${ }^{69}$ ) These results demonstrate that the effect of the field due to the silica atoms is important. Specifically, the frequency distribution obtained by completely neglecting the contributions of the silica atoms to the electric field has a strong shoulder to the high-frequency side of the distribution that is absent in bulk water and the results from the other three methods. Interestingly, the frequency distribution is not highly sensitive to the details of how its calculation is implemented: whether the silica charges are scaled, only included within a cut-off region, or included for

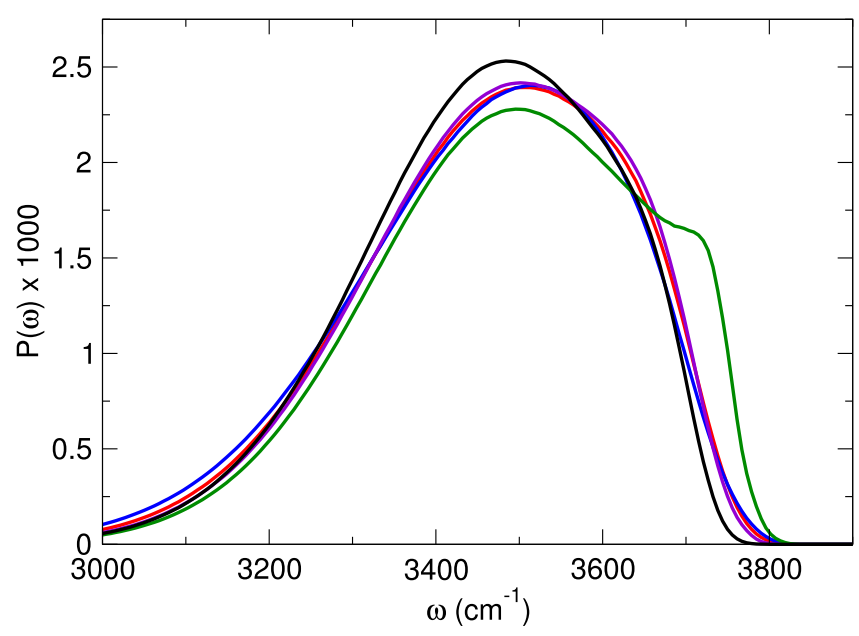

FIG. 2. Simulated distribution of empirical mapping-based $\mathrm{OH} n=0 \rightarrow n$ $=1$ frequencies for bulk water (black) and water confined in a $\sim 2.4 \mathrm{~nm}$ hydrophilic silica pore. Results are shown for the electric field calculated using a distance cut-off (red), a distance cut-off with scaled silica charges (blue), no distance cut-off for silica atoms (violet), and neglecting silica contributions to the field (green). (Results are from a single 1 ns trajectory.)

all atoms making up the pore does not strongly affect the frequency distribution. The biggest differences occur when scaling the charges, which leads to less blue-shifting of the $\mathrm{OH}$ frequencies such that the distribution does not extend as far to higher frequencies and has a slightly larger tail at lower frequencies. However, all three methods for incorporating the field due to the silica atoms give the same semi-quantitative results and the same trends relative to the distribution for bulk water. For this reason, in the following, we calculate the field due to the silica pore atoms by including any atom within a distance $R_{\text {cut }}$ of the $\mathrm{H}$ atom of interest (the $\mathrm{O}$ and $\mathrm{H}$ atoms of silanol groups are always included together).

\section{SIMULATED VIBRATIONAL SPECTRA}

\section{A. Frequency distributions}

With the approach adopted for calculating the $n=0$ $\rightarrow n=1 \mathrm{OH}$ stretching vibrational frequencies as described in Sec. II A, we can consider the comparison between the bulk and confined water frequency distributions as shown in Fig. 2. The bulk water distribution has an asymmetric shape, decaying faster at large $\omega$ and more slowly as $\omega$ decreases. It reaches a maximum at $3484 \mathrm{~cm}^{-1}$ while the average frequency is lower at $\langle\omega\rangle=3456 \mathrm{~cm}^{-1}$. The key difference between the bulk and the confined water frequency distributions is that the latter is shifted to higher frequencies. The confined water distribution peaks around $3510 \mathrm{~cm}^{-1}$, but the somewhat broader distribution of frequencies gives an average frequency of $3463 \mathrm{~cm}^{-1}$, only $7 \mathrm{~cm}^{-1}$ higher than in the bulk.

The distribution of frequencies can be decomposed into the contributions from $\mathrm{OH}$ moieties at different distances from the interface. Here we use the distance, $d$, of the $\mathrm{H}$ atom of the hydroxyl group to the nearest pore oxygen atom to quantify the location. The distance-resolved distributions are shown in Fig. 3. It is clear from this data that the blue-shifted nature of the confined water spectrum, relative to that of bulk water, 

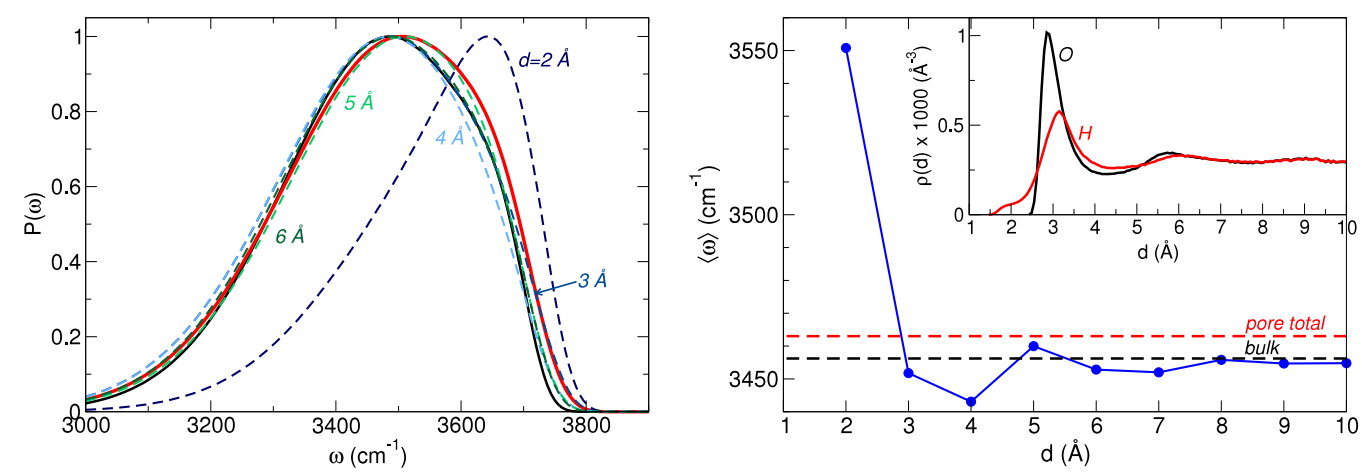

FIG. 3. Left: Simulated distribution of $\mathrm{OH} n=0 \rightarrow n=1$ frequencies for bulk (black) and confined (red) water as in Fig. 2. The frequency distributions for OH groups at different distances from the pore surface are also shown. Here, $d$ is the distance of the $\mathrm{H}$ atom to the nearest pore oxygen atom. The distributions for $d \geq 6 \AA$ (not shown) are indistinguishable from each other and the bulk water distribution. Distributions are normalized so the maximum is 1. Right: Average $n=0 \rightarrow n=1$ frequency is plotted versus distance, $d$, from the pore wall; values for bulk and all confined water are shown for comparison. Inset shows the atomic densities as a function of $d$ for water oxygen $(\mathrm{O})$ and hydrogen $(\mathrm{H})$ atoms.

is due to waters near the silica pore interface. In particular, the frequency distribution for $d=2 \AA$ peaks at significantly higher frequencies than the bulk distribution as well as that for all other distances. Indeed, the $d=2 \AA$ distribution has an average frequency of $3551 \mathrm{~cm}^{-1}$, as shown in Fig. 3, which is approximately $100 \mathrm{~cm}^{-1}$ higher than the bulk and all $d \geq 3 \AA$. The distribution for $d=2 \AA$ is also significantly narrower than that for all other distances, but with a tail to lower frequencies. This population corresponds primarily to $\mathrm{OH}$ groups that are H-bond donors to the pore surface, and the blueshift of the frequency is due to the weaker H-bonds formed with pore oxygen atoms. As the distance from the pore surface increases, the frequency distribution quickly approaches that of the bulk liquid. The distribution for $d=3 \AA$ is similar to that of bulk water but slightly broader, particularly on the higher-frequency side. At slightly larger distances, $d=4$ and $5 \AA$, the frequency distributions are quite similar to the bulk, but with slight variations in the shape of the distribution on the blue side of the distribution. Interestingly, the convergence to the bulk distribution is non-monotonic as the $d=4 \AA$ case has a diminished probability and the $d=5 \AA$ has an enhanced probability to the blue, though these are small effects.

Additional insight into the location-dependence of the $\mathrm{OH}$ frequency can be gained by examining the average frequency as a function of the distance $d$. These data are shown in Fig. 3 . Clearly, consistent with the full distributions, these results show that the shift in average frequency for the pore is due exclusively to $\mathrm{OH}$ groups that are within $3 \AA$ of the surface and dominated by those that are within $2 \AA$, i.e., close enough to $\mathrm{H}$-bond with the pore surface. Indeed, the average frequency for $d=2 \AA$ is $\sim 100 \mathrm{~cm}^{-1}$ blue-shifted relative to all distances further from the interface. Except for $d=4 \AA$, for which $\left\langle\omega_{01}\right\rangle$ is $13 \mathrm{~cm}^{-1}$ lower than the bulk, all distances give average frequencies within $5 \mathrm{~cm}^{-1}$ of the bulk value. As noted above, there is a non-monotonic behavior in the distance-dependent distributions that is notable between $d=4-5 \AA$. Some insight into this behavior is given by the inset in Fig. 3, which shows the density for water oxygen and hydrogen atoms in the pore as a function of the distance $d$. The hydrogen density shows a minimum near $\sim 4.4 \AA$, following the interfacial layer. Thus, the frequency distribution for $d=4 \AA$ is dominated by $\mathrm{OH}$ groups engaged in slightly stronger $\mathrm{H}$-bonds due to the liquid layering; in contrast, the $d=5 \AA$ frequency distribution arises primarily from OHs just outside the interfacial layer that are, on average, donating slightly weaker H-bonds. There is little effect from the interface after $d \sim 6-7 \AA$ (and no effect for $d \geq 8 \AA$ ) observed in either the liquid layering, as shown in the density, $\rho(d)$, or in the average frequency, $\langle\omega\rangle$.

\section{B. Infrared (IR) lineshapes}

The frequency mapping approach can be used to predict the infrared lineshapes of confined water as well. The IR lineshape can be calculated as the Fourier transform,

$$
I(\omega)=\frac{1}{2 \pi} \int_{-\infty}^{\infty} e^{-i(\omega-\langle\omega\rangle) t} \phi(t) d t,
$$

where $\phi(t)$ is the dipole-dipole response function,

$$
\phi(t)=\left\langle\vec{\mu}_{01}(0) \cdot \vec{\mu}_{01}(t) e^{i \int_{0}^{t} \delta \omega(\tau) d \tau}\right\rangle e^{-|t| / 2 T_{1}} .
$$

Here, $\delta \omega(t) \equiv \omega(t)-\langle\omega\rangle, \vec{\mu}_{01}(t)=\mu_{01}(t) \overrightarrow{\mathbf{e}}_{O H}(t)$ is the $0 \rightarrow 1$ (fundamental) transition dipole vector for the $\mathrm{OH}$ oscillator directed along the bond, and $T_{1}$ is the vibrational energy relaxation time. In this work, $T_{1}$ is taken from experiment as $700 \mathrm{fs} .{ }^{70}$ As with the transition frequency, the transition dipole magnitude, $\mu_{01}$, including non-Condon effects, is determined within the empirical mapping approach. ${ }^{64,65}$

The IR lineshape for bulk water and water in the $\sim 2.4 \mathrm{~nm}$ diameter silica pore are shown in Fig. 4. These results show that nanoscale confinement has a surprisingly small effect on the IR spectrum. Confined water has an IR lineshape that is centered around $3400 \mathrm{~cm}^{-1}$, as is that for the bulk liquid. The central difference is that the IR lineshape for water in the silica pore is slightly broader. This broadening is distinctly different than that observed for the frequency distribution shown in Figs. 2 and 3, which is broader principally on the higher-frequency side. The IR lineshape is broader to the blue and red sides of the spectrum and shows no clear hint of this addition of higher frequency $\mathrm{OH}$ modes associated with the interfacial waters and observed in the frequency distribution.

This surprisingly small effect on the IR lineshape upon confinement has been previously observed in measurements. ${ }^{23,25,26}$ Musat et al. found little change from the bulk 

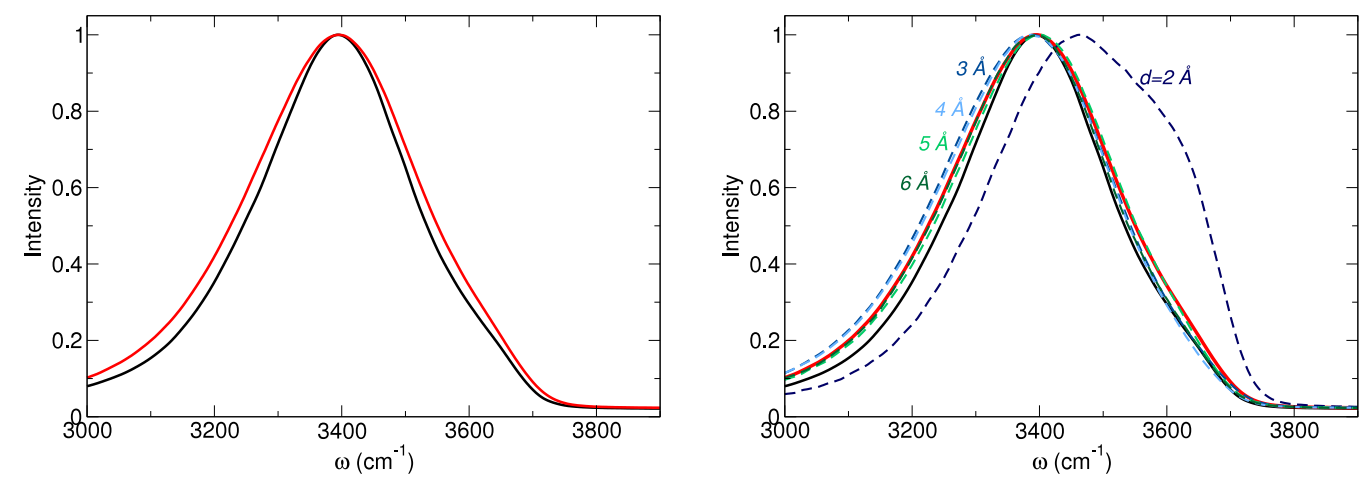

FIG. 4. Left: Simulated linear IR spectrum of the $\mathrm{OH}$ fundamental for bulk water (black) and water confined in a $\sim 2.4$ nm hydrophilic silica pore (red). Right: Spectra for $\mathrm{OH}$ groups at different distances, $d$, from the pore surface are shown. Spectra for $d \geq 6 \AA$ are indistinguishable from each other and the bulk water distribution. All spectra are scaled so that the maximum value is 1 .

spectrum when water was confined to 50,13 , and even $1 \mathrm{~nm}$ diameter pores of controlled pore glasses, ${ }^{23}$ which is in accord with the present simulations. Further, they measured the spectrum for "low hydration" water in $1 \mathrm{~nm}$ pores, involving only interfacial water. That spectrum is significantly broadened relative to the bulk and fully-filled pore spectra with a distinct blue-shifted shoulder. This is generally consistent with the blue-shifted frequencies of the interfacial water observed in the calculations (with the broadening presumably due to both greater inhomogeneous broadening and reduced motional narrowing for this low hydration water).

It is interesting to note that these results are quantitatively different than what is observed in (AOT and Igepal) reverse micelles $5,17,20,22,29,30,71-73$ where the $\mathrm{OH}$ (or OD) spectra are found to shift consistently to higher frequencies with increasing confinement (smaller reverse micelles). Fayer and co-workers have shown that this result can be understood in terms of a "shell" of interfacial water that exhibits blueshifted frequencies combined with a "core" waters away from the interface with a spectrum like that of bulk water. ${ }^{5,17,20,22}$ Reducing the reverse micelle size increases the fraction of shell water molecules, leading to the consistent blue-shift. In contrast with the silica pores considered here, these $\mathrm{OH}$ groups with blue-shifted frequencies are readily observable in the IR spectra. Simulations indicate that non-Condon effects are still important for these interfacial waters (though perhaps quantitatively different than in mesoporous silica) and thus the difference between the systems is likely attributable to the greater fraction of blue-shifted $\mathrm{OHs}$ contributing in spherical reverse micelles compared to cylindrical silica pores. ${ }^{29,30}$

The distance-dependent IR lineshapes are shown in Fig. 4(b); the distance is determined by the hydrogen atom distance, $d$, at time $t=0$ in the calculation of the time correlation function in Eq. (5). As with the frequency distribution, the IR lineshape for $\mathrm{OH}$ groups with the hydrogen $2 \AA$ from a pore oxygen atom is strongly blue-shifted relative to the bulk liquid and all other distances. The lineshapes for $\mathrm{OH}$ groups further from the surface are quite close to that of the bulk spectrum, though even at $6 \AA$ the lineshape is not fully converged to the bulk result. The same slight nonmonotonic convergence towards the bulk lineshape found for the frequency distribution is observed for distances between 3 and $5 \AA$.
Insight into the relative invariance of the IR lineshape with confinement can be obtained by examining the spectral density. Namely, the relative role of non-Condon and dynamical effects in determining the lineshape can be evaluated by comparing the full lineshape to the frequency distribution weighted by the transition dipole moment squared, i.e., the spectral density,

$$
P_{\mu}(\omega)=\left\langle\mu_{01}^{2} \delta\left(\omega-\omega_{01}\right)\right\rangle .
$$

This spectral density is shown in Fig. 5 where it is compared to the IR lineshape and the unweighted frequency distribution. Notably, the spectral density is quite distinct from the frequency distribution, but it is quite similar to the IR lineshape, differing principally in a small redshift of the peak position and a broader shape. The peak position of the spectral density is red-shifted by $<10 \mathrm{~cm}^{-1}$ from that of the IR spectrum, while the frequency distribution is blueshifted by $\sim 110 \mathrm{~cm}^{-1}$; the full-width half maximum (FWHM) of the IR spectrum is $\sim 326 \mathrm{~cm}^{-1}$ compared to 390 and $410 \mathrm{~cm}^{-1}$ for the spectral density and frequency distribution, respectively. The similarity of the spectral density and the IR lineshape suggests that it is non-Condon effects, i.e.,

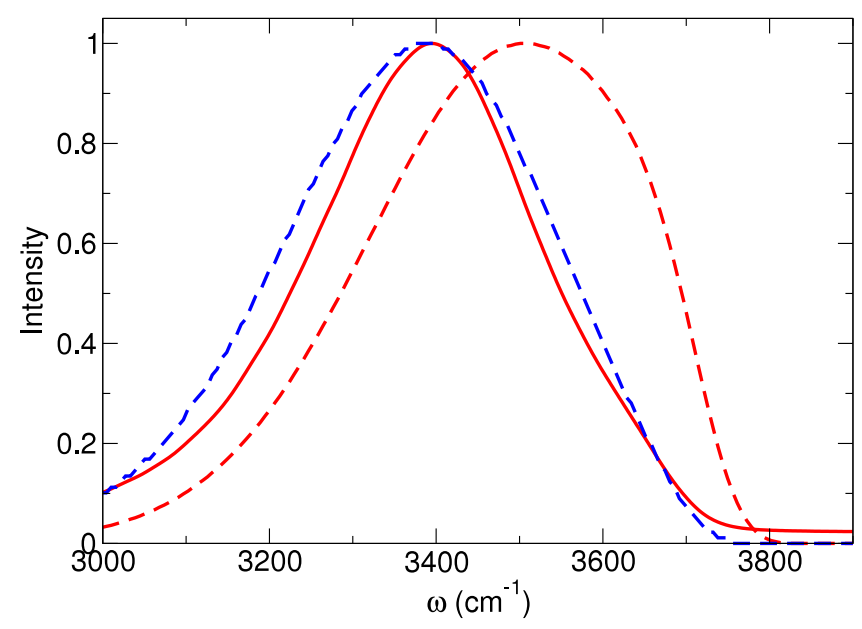

FIG. 5. Simulated linear IR spectrum, $I(\omega)$, Eq. (4), of water confined in a $\sim 2.4 \mathrm{~nm}$ hydrophilic silica pore (solid red) is compared to the corresponding frequency distribution, $P(\omega)$, (dashed red), Eq. (3), and spectral density, $P_{\mu}(\omega)$ (dashed blue), Eq. (6). 
the environment-dependent transition dipole moments, that are principally responsible for the shape of the latter. In particular, the contribution to the IR spectrum of the more weakly $\mathrm{H}$-bonded, blue-shifted, $\mathrm{OH}$ groups near the silica pore surface are suppressed due to their smaller transition dipole moments. At the same time, the difference in the FWHM of the IR lineshape and spectral density suggest some role for dynamics. As can be seen from Fig. 4, the confined $\mathrm{HOD} / \mathrm{D}_{2} \mathrm{O}$ spectrum is slightly broader than that for the bulk liquid. The similarities in the frequency distributions indicates at best a small role for greater inhomogeneous broadening and points instead to reduced motional narrowing of the spectrum as the most likely explanation for the larger IR linewidth in the confined liquid. Additional evidence for this reduced motional narrowing for confined water is provided in Sec. III E.

\section{Raman spectra}

The Raman lineshape can be obtained in an analogous fashion to the infrared spectrum as the Fourier transform of a response function. Here, we focus on the isotropic Raman spectrum such that

$$
I_{i s o}(\omega)=\frac{1}{2 \pi} \int_{-\infty}^{\infty} e^{-i(\omega-\langle\omega\rangle) t} \phi_{i s o}(t) d t,
$$

where

$$
\phi_{\text {iso }}(t)=\left\langle\alpha_{01}(0) \alpha_{01}(t) e^{i \int_{0}^{t} \delta \omega(\tau) d \tau}\right\rangle e^{-|t| / 2 T_{1}},
$$

and $\alpha_{01}$ is the transition polarizability between the ground and first-excited vibrational states and the remaining variables are as described in Sec. III B. For the isotropic lineshape, this polarizability is $\alpha=\left(\alpha_{x x}+\alpha_{y y}+\alpha_{z z}\right) / 3 .^{74}$

The isotropic Raman lineshapes calculated in this way are shown in Fig. 6 for bulk water and silica-confined water. A distinct difference is observed between the two results. The bulk water Raman spectrum peaks around $3442 \mathrm{~cm}^{-1}$ and exhibits a significant shoulder to the blue side, located near $3625 \mathrm{~cm}^{-1}$. The same general features are found in the Raman spectrum of water in the silica pore, with some key differences. For confined water, the peak position is shifted to the blue by $\sim 10 \mathrm{~cm}^{-1}$ relative to the bulk liquid, the spectrum is broader (bulk FWHM $\simeq 369 \mathrm{~cm}^{-1}$, confined FWHM $\simeq 395 \mathrm{~cm}^{-1}$ ), and, most prominently, the higher-frequency side of the spectrum, including the shoulder, has significantly greater intensity. In this way, the Raman spectrum is more similar to the frequency distribution than the IR spectrum. This is expected as it is now well known that non-Condon effects, which suppress the blue-shifted frequencies due to weaker H-bonds in the IR spectrum, are considerably smaller in the Raman spectrum. ${ }^{74}$ This indicates that the effect of confinement on the water $\mathrm{OH}$ oscillators is more clearly observed in the Raman spectrum than the IR spectrum.

The simulated lineshapes are in general agreement with experimental Raman spectra reported in the literature. ${ }^{24,75,76}$ For example, Huang et al. measured the Raman spectrum of $\mathrm{H}_{2} \mathrm{O}$ confined in $6.9 \mathrm{~nm}$ diameter Vycor glass and compared it to the bulk water spectrum. ${ }^{24}$ They found that the key differences between bulk and confined water are a general blue-shifting of the spectrum along with the appearance of a shoulder on the high-frequency side. They also observed a decrease in the red-shifted shoulder around $3200 \mathrm{~cm}^{-1}$ that appears in the neat $\mathrm{H}_{2} \mathrm{O}$ spectrum, ${ }^{77,78}$ but is absent in our simulated spectra for $\mathrm{HOD} / \mathrm{D}_{2} \mathrm{O}$.

More insight into the Raman spectrum can be obtained from the distance-dependent lineshapes, which are also shown in Fig. 6. The results are qualitatively similar to those for the frequency distribution shown in Fig. 3. Namely, the Raman lineshape for $\mathrm{OH}$ groups nearest the pore surface, $d=2 \AA$, are significantly blue-shifted relative to bulk water and all other distances. Indeed, the $d=2 \AA$ spectrum peaks near $3645 \mathrm{~cm}^{-1}$, approximately $200 \mathrm{~cm}^{-1}$ higher in frequency than the bulk spectrum and the total spectrum for the confined water, but close to the position of the blue-shifted shoulder that appears in both the bulk and confined water spectra, but is more prominent in the latter. The lineshapes for all other distances exhibit peaks around $3440-3450 \mathrm{~cm}^{-1}$, close to that of the bulk liquid. The spectra for $d \geq 3 \AA$ are broader than the bulk water spectrum but converge to it with increasing $d$; as with the frequency distribution and IR lineshape, this convergence is non-monotonic with the $d=4 \AA$ lineshape more redshifted and narrower than $d \geq 5 \AA$. The $d=6 \AA$ lineshape is quite close to that of the bulk liquid, with only some small, quantitative differences. However, the $d=6 \AA$ spectrum is nearly the same as that for larger distances (not shown) indicating that, while water in the pore interior exhibits a lineshape quite close to bulk water, the bulk limit is not quantitatively observed.
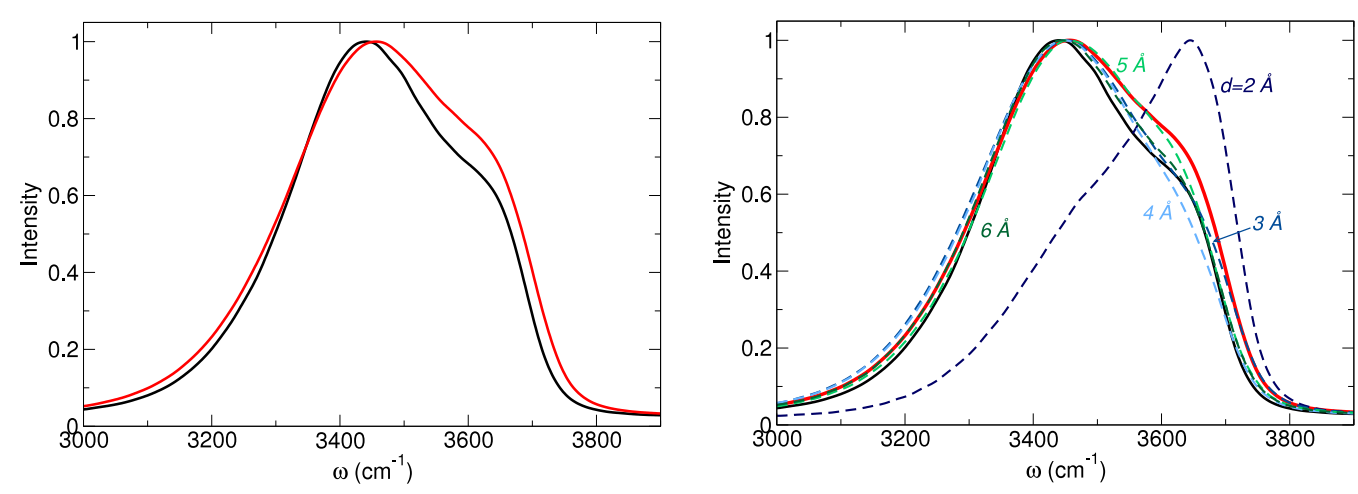

FIG. 6. Left: Simulated isotropic Raman lineshapes of the $\mathrm{OH}$ fundamental for bulk water (black) and water confined in a $\sim 2.4 \mathrm{~nm}$ hydrophilic silica pore (red). Right: Spectra for $\mathrm{OH}$ groups at different distances, $d$, from the pore surface are shown. All spectra are scaled so that the maximum value is 1 . 


\section{2D-IR photon echo spectra}

The two-dimensional infrared spectra are calculated from the third-order nonlinear response functions as described in Ref. 79. The results are shown in Fig. 7 for different waiting times, $T_{w}$, from 0 to 6 ps for both the bulk $\mathrm{HOD} / \mathrm{D}_{2} \mathrm{O}$ and the liquid confined in a silica pore. In brief, the 2D-IR spectra probe the correlation between the initial frequency of a given $\mathrm{OH}$ oscillator (the horizontal, $\omega_{1}$, axis) and its frequency after evolving in the liquid environment for a time $T_{w}$ (the vertical, $\omega_{3}$, axis). Thus, the spectra have peaks at early times, e.g., $T_{w}=0 \mathrm{fs}$, that show a strong positive correlation between $\omega_{1}$ and $\omega_{3}$. (Note that two peaks are present, a positive peak at higher frequencies associated with the $v=0 \rightarrow 1$ transition (colored yellow-red) and a negative peak at lower frequencies associated with the $v=1 \rightarrow 2$ transition (colored blue).) As the waiting time increases, this correlation decreases leading to more rounded, isotropic peak shapes. Thus, the 2D-IR spectra report, among other things, on how long it takes an $\mathrm{OH}$ bond to lose memory of a given frequency through interaction with its environment.

It is clear from the spectra presented in Fig. 7 that there is a difference between these dynamics in bulk $\mathrm{HOD} / \mathrm{D}_{2} \mathrm{O}$ compared to the confined liquid. In particular, the loss of correlation between the $\omega_{1}$ and $\omega_{3}$ is nearly complete in roughly 2 ps for bulk water, as indicated by the appearance of nearly isotropic peak shapes. In contrast, $\mathrm{HOD} / \mathrm{D}_{2} \mathrm{O}$ in a silica pore retains this correlation for significantly longer, even out to $6 \mathrm{ps}$. This qualitative trend in the decay of the frequency correlation is expected as the rotational and diffusional dynamics of water in silica pores is significantly slowed compared to the bulk liquid. ${ }^{39,46,47}$ Moreover, these results suggest that these slower dynamics should be observable in measurements of the 2D-IR spectra of confined water, which have not yet been carried out. The quantitative differences are explored in greater detail in Sec. III E below.

The results for the linear IR and Raman spectra shown above indicate that it is the $\mathrm{OH}$ groups nearest to the interface, indeed those that are close enough to donate an H-bond to a pore oxygen atom, that exhibit strongly modified vibrational frequencies. It is then interesting to examine the contribution to the 2D-IR spectrum as a function of $\mathrm{OH}$ position. These results are presented in Fig. 8, where the spectra for $T_{w}=0$ to $1 \mathrm{ps}$ are shown for $\mathrm{OH}$ groups that, at time $t=0$ in the calculation of the response functions, are at a given distance $d$ from the nearest pore oxygen atom. The bulk 2D-IR spectra are shown for comparison. The spectra clearly show that those molecules closest to the pore interface, $d=2 \AA$, are indeed the most strongly affected by the confinement. There is little loss of the frequency correlation in the $d=2 \AA$ spectra even out to $1 \mathrm{ps}$ and the peak is significantly blue-shifted, as observed for the linear IR spectrum for $d=2 \AA$ shown in Fig. 4. However,
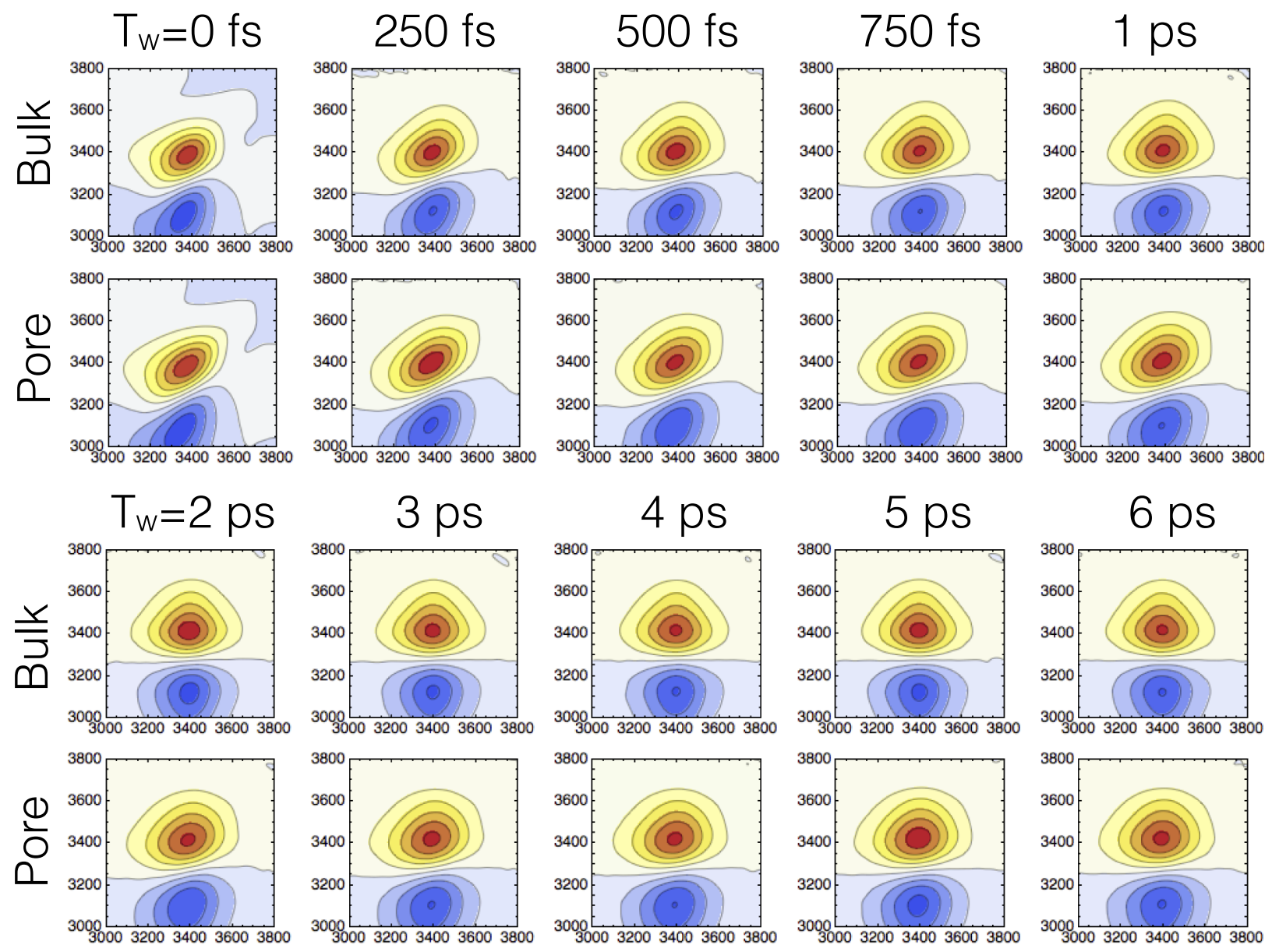

FIG. 7. Simulated 2D-IR photon echo spectra of $\mathrm{HOD} / \mathrm{D}_{2} \mathrm{O}$ in the bulk liquid and confined in a $\sim 2.4 \mathrm{~nm}$ silica pore. The horizontal axis is $\omega_{1}$ and the vertical $\omega_{3}$. For each waiting time, $T_{w}, 10$ contours are shown at even intervals between $120 \%$ of the minimum and maximum intensities. 


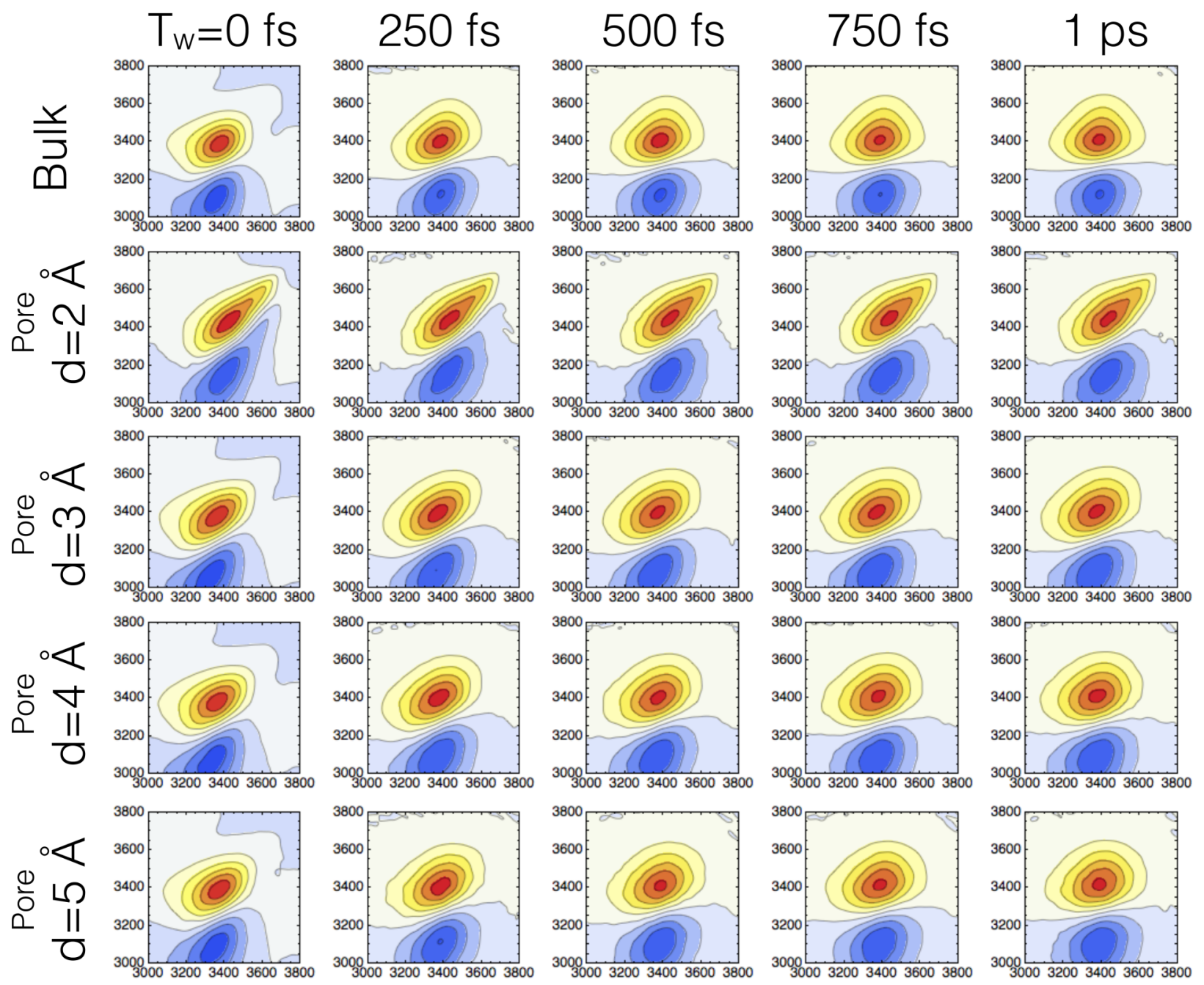

FIG. 8. Same as Fig. 7 except that the pore spectra are shown for positions relative to the pore surface. The signal is obtained by including OH groups at the distance, $d$, at $t=0$ in the calculation of the response function.

the contribution of the $\mathrm{OH}$ groups this close to the pore surface to the total 2D-IR spectrum is limited both by their comparatively small number and the non-Condon effects, i.e., the smaller transition dipole moment for these more weakly H-bonded groups.

The loss of frequency correlation occurs more rapidly at larger distances from the pore interface, with a significant, qualitative change between $d=2$ and $3 \AA$, similar to what was observed for the $\mathrm{OH}$ frequencies and linear spectra. For $d=3-5 \AA$, the $\mathrm{OH}$ groups lose memory of their frequency on time scales longer than that of the bulk liquid, but more rapidly with increasing $d$. It is interesting that these $\mathrm{OH}$ frequency dynamics are still slower than the bulk liquid for $d=5 \AA$, even though the distribution of frequencies and the linear IR spectrum for these $\mathrm{OH}$ groups are quite similar to the bulk.

It is useful to examine features of the 2D-IR spectra more quantitatively. One such approach is through estimates of the homogeneous and inhomogeneous linewidths, ${ }^{80}$ which can be approximately extracted from the 2D-IR spectrum as the linewidth along the anti-diagonal and diagonal, respectively. Due to the different origins of the two linewidths, the homogeneous linewidth, determined by the timescales of frequency fluctuations, is determined by fitting to a Lorentzian, while the inhomogeneous linewidth, due to the distribution of static frequencies, is extracted from a Gaussian fit. The results are shown as a function of waiting time in Fig. 9 for both the confined and bulk liquids. The results show that, for both the bulk and confined $\mathrm{HOD} / \mathrm{D}_{2} \mathrm{O}$, the homogeneous linewidths increase and the inhomogeneous linewidths decrease with increasing waiting time. As expected, for each system the homogeneous and inhomogeneous linewidths converge to the same value at long waiting times. At short times confinement has little effect on the homogeneous linewidth which is dominated by frequency dynamics associated with librational motion. At longer waiting times, the confined $\mathrm{HOD} / \mathrm{D}_{2} \mathrm{O}$ homogeneous linewidth is larger than the corresponding bulk value, presumably due to both the wider range of environments sampled and the reduced motional narrowing due to slower frequency dynamics (see Sec. III E). The inhomogeneous linewidth decreases with time but the linewidth for the confined liquid is $\sim 15 \%-20 \%$ larger than that for the bulk liquid. This modest trend is expected from the broader distribution of frequencies for silica-confined $\mathrm{HOD} / \mathrm{D}_{2} \mathrm{O}$ due to the weaker $\mathrm{H}$-bonds between interfacial waters and silica oxygens. 


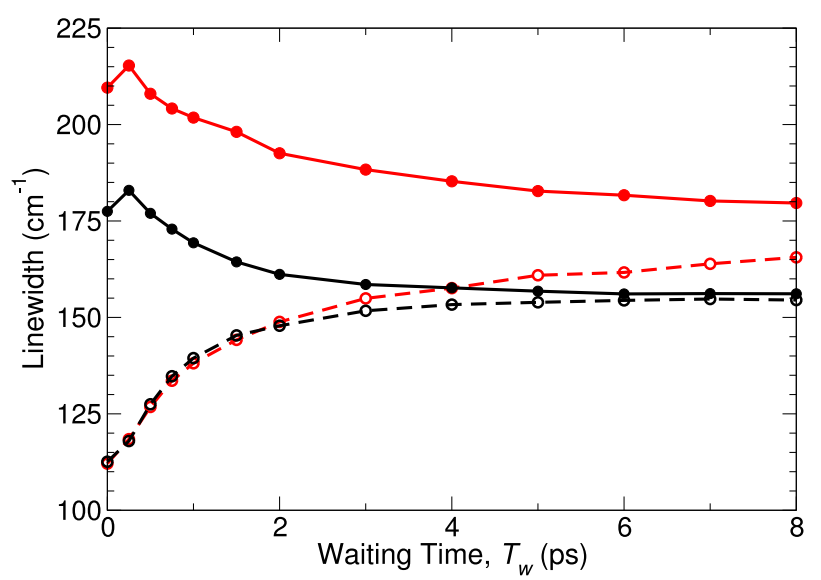

FIG. 9. Inhomogeneous (solid lines) and homogeneous (dashed lines) linewidths are plotted as a function of waiting time. Results are shown for both bulk (black) and confined (red) $\mathrm{HOD} / \mathrm{D}_{2} \mathrm{O}$.

\section{E. Spectral diffusion}

The frequency dynamics of the $\mathrm{OH}$ groups can also be examined by more quantitative measures. One method for doing so is the center-line-slope (CLS) approach. ${ }^{81}$ The center-line is defined by connecting the value of $\omega_{3}$ for which the positive-going, $v=0 \rightarrow 1$ peak in the $2 \mathrm{D}$-IR spectrum is a maximum for each value of $\omega_{1}$. The slope is then obtained by fitting this line in a region near the peak maximum, giving a measure of the correlation between the initial $\left(\omega_{1}\right)$ and final $\left(\omega_{3}\right)$ frequencies. We have calculated this over a $150 \mathrm{~cm}^{-1}$ range of $\omega_{1}$ values centered on the $0 \rightarrow 1$ peak position. The results for both bulk and confined $\mathrm{HOD} / \mathrm{D}_{2} \mathrm{O}$ are shown in Fig. 10 as a function of waiting times, $T_{w}$.

Kwak et al. ${ }^{81}$ showed that under certain conditions the CLS is equal to the frequency autocorrelation function,

$$
C_{\omega}(t)=\left\langle\delta \omega_{01}(0) \delta \omega_{01}(t)\right\rangle /\left\langle\delta \omega_{01}(0)^{2}\right\rangle,
$$

where $\delta \omega_{01}(t) \equiv \omega_{01}(t)-\left\langle\omega_{01}\right\rangle$. This correlation function explicitly measures the loss of memory of the transition frequency for $\mathrm{OH}$ groups, i.e., the spectral diffusion, and can be conveniently calculated from simulations. Thus, the calculated $C_{\omega}(t)$ is compared with the CLS results in Fig. 10 to test this connection. The results show that the CLS from the simulated 2D-IR spectra are in good agreement with the calculated frequency autocorrelation function. This indicates that the CLS can still be a faithful representation of spectral diffusion for confined liquids (even though the assumption of a single, homogeneous population made in the derivation in Ref. 81 is not met here). The results themselves show that, as anticipated by the qualitative discussion of the 2D-IR spectra above, the $\mathrm{OH}$ groups in silica-confined $\mathrm{HOD} / \mathrm{D}_{2} \mathrm{O}$ lose memory of their frequency more slowly that those in the bulk liquid. Moreover, the difference, while not as extreme as observed for reorientational dynamics, ${ }^{39,46,47}$ are significant and should be measurable. The bulk CLS is well described by an exponential decay with a timescale of $1.1 \mathrm{ps}$. The pore CLS exhibits bi-exponential behavior and can be fit with one timescale that matches that of the bulk liquid and another that is more than $5 \mathrm{ps}$ (precise determination of this timescale is challenging due to the limited waiting times that can be reasonably accessed).

More detailed analysis of the spectral diffusion can be carried out using the frequency autocorrelation function. This $C_{\omega}(t)$ is shown for bulk water and silica-confined water in Fig. 10 along with the results restricted by initial $(t=0)$ distance of the $\mathrm{OH}$ group. The frequency time correlation function decays with a timescale that represents the loss of memory of the transition frequency and in both bulk and confined water is best described by three timescales that can be attributed to inertial dynamics ( $\tau_{\text {iner }} \sim 30-40 \mathrm{fs}$ ), librational dynamics $\left(\tau_{l i b r} \sim 300 \mathrm{fs}\right)$, and a longer timescale for spectral diffusion, $\tau_{\omega}$, related to H-bond breaking and making. This last timescale is approximately $1.2 \mathrm{ps}$ for bulk water, in agreement with the CLS results. In contrast, for confined water, the librational timescale is somewhat longer, but the longest decay is at least five times slower than in bulk water, again consistent with the CLS data. This spectral diffusion is also distance dependent, as would be expected given the frequency shifts observed for water molecules at the silica interface. The $\tau_{\omega}$ timescales are slowest for $d=2 \AA$, for which it is $>10 \mathrm{ps}$, and decreases with increasing distance from the pore wall to $\sim 6$ ps for $d=6 \AA$. The change in $\tau_{\omega}$ is greatest between $d=2$ and $3 \AA$, however, and is only weakly dependent on distance for larger $d$. This indicates that while the distribution of frequencies, shown in Fig. 3, is quite similar to that of the bulk liquid for $d \geq 3 \AA$, the spectral
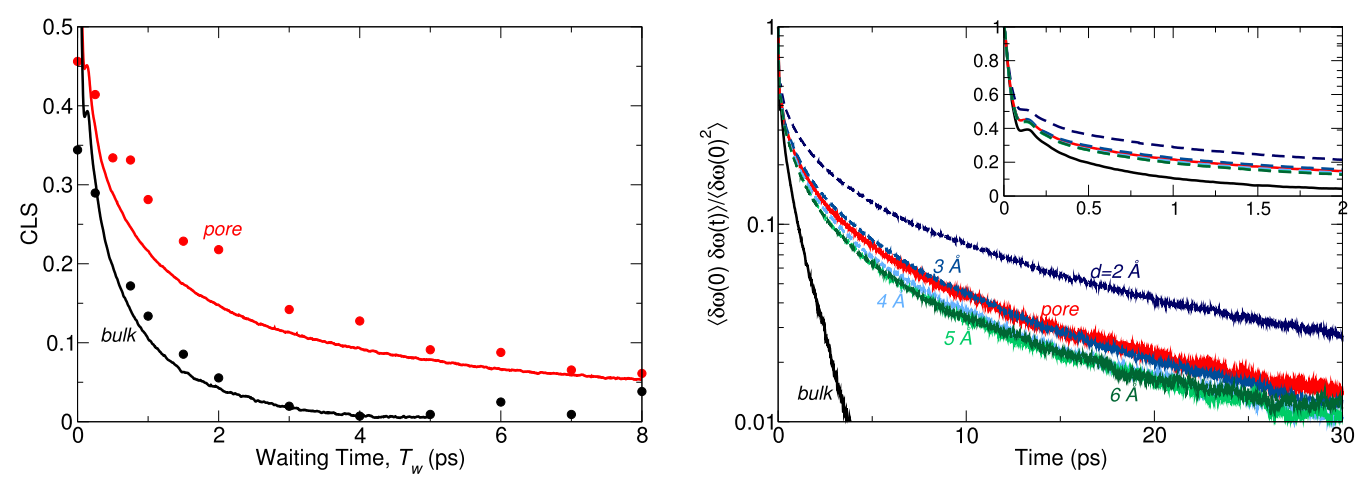

FIG. 10. Left: Center-line-slope (filled circles) is shown as a function of waiting time and compared to the normalized frequency autocorrelation function (solid lines), Eq. (9). Right: Normalized frequency autocorrelation function for bulk water (black), water confined in a silica pore (red), and confined water distinguished by the distance, $d$, from the pore wall at time $t=0$. The correlation functions for $d \geq 6 \AA$ are indistinguishable. 

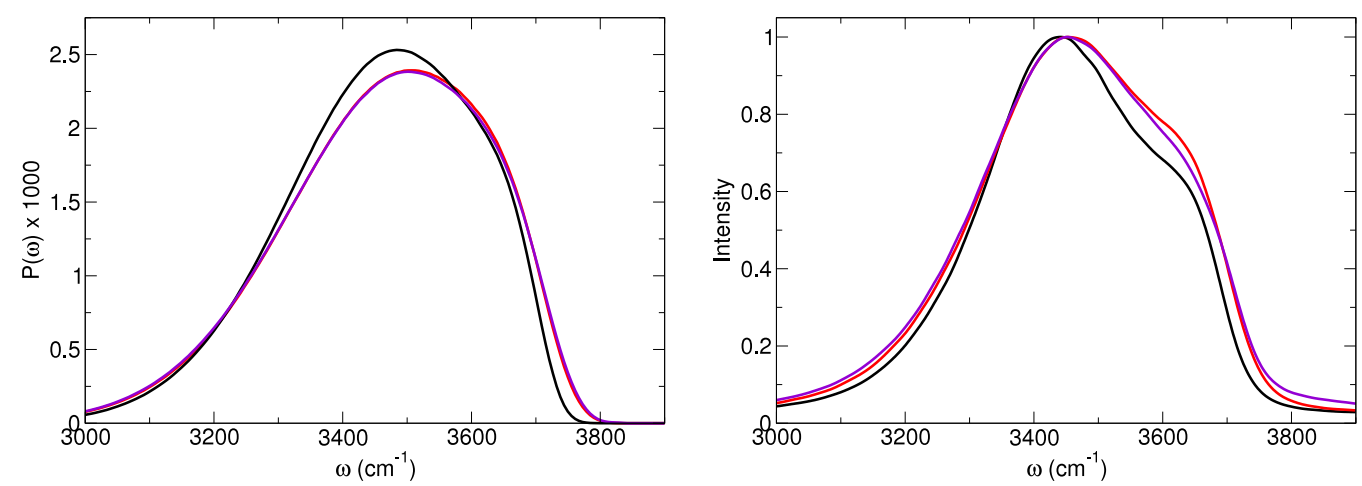

FIG. 11. Left: Frequency distribution, $P(\omega)$, for the $\mathrm{OH}$ fundamental for bulk $\mathrm{HOD} / \mathrm{D}_{2} \mathrm{O}$ (black) and $\mathrm{HOD} / \mathrm{D}_{2} \mathrm{O}$ confined in two different $\sim 2.4 \mathrm{~nm}$ hydrophilic silica pores, pore 1 (red) with 56 surface silanols and pore 2 (violet) with 86 surface silanols. Right: Simulated isotropic Raman spectra for the same systems.

diffusion is more strongly affected and slowed throughout the pore.

It is interesting to compare these timescales with those obtained in a previous detailed examination of H-bonding of water in the same silica pores. ${ }^{47}$ While the dynamics for exchange of H-bond partners was found to be inhomogeneous and site-dependent, the average time for an $\mathrm{OH}$ group to switch from an H-bond with a silica acceptor to a new water acceptor is $\sim 5-6$ ps. $^{47}$ Thus, the longer timescale observed in the CLS is consistent with that for H-bond exchange from a weaker silica oxygen acceptor (where the $\mathrm{OH}$ frequency is blue-shifted) to a water oxygen (where the average $\mathrm{OH}$ frequency is closer to the bulk value).

\section{F. Effects of pore structure}

To probe the effects of the specific pore structure on the spectral features, simulations were performed on a second pore model with the same nominal radius but different silanol density. As described in Sec. II, the two pores were generated by the same procedure but different initial conditions, ${ }^{31}$ such that the amorphous structures differ in the atomic-level details. Because the functionalization of the pore surface is based on the $\mathrm{Si}-\mathrm{O}$ bond distances, pore 1 , from which the results reported in the preceding sections were obtained, has 56 silanol ( $\mathrm{Si}-\mathrm{OH}$ ) groups, while pore 2 has 86 ; these correspond to 2.5 and $3.8 \mathrm{OH} / \mathrm{nm}^{2}$, respectively. Thus, comparison of spectra from simulations of the two pores can provide some insight into general effects of heterogeneity as well as silanol density.

The frequency distributions and isotropic Raman spectra for the two pores are compared in Fig. 11 along with the bulk liquid results. The frequency distributions calculated from the two pores are nearly identical, with the peak position and width effectively the same. The only distinction that is observed is that pore 2 exhibits a slightly slower decay to high frequencies compared to pore 1 , but the difference is barely outside the width of the lines. The IR spectra for the two pores, shown in Fig. S2 of the supplementary material, ${ }^{69}$ are also essentially identical. The same is true for the 2D-IR photon echo spectra which are plotted in Fig. S3 and show no clear differences between the two pores. ${ }^{69}$ The spectral dynamics that underlie these nonlinear IR spectra can be examined independently by comparing the frequency autocorrelation functions in the two pores. This is done in Fig. S4, ${ }^{69}$ which shows $C_{\omega}(t)$ for pore 2 compared to that for pore 1 (averaged over all five $1 \mathrm{~ns}$ trajectories and for each individual trajectory). The results show that the spectral dynamics are not strongly sensitive to the pore surface silanol density or atomistic structure.

The isotropic Raman spectra do show noticeable differences between the two pores, however. The key effect is seen in the blue-shifted shoulder, where the pore 2 spectrum has slightly less intensity around $3650 \mathrm{~cm}^{-1}$ compared to the pore 1 spectrum. This difference is similar to that in the frequency distribution but with a larger magnitude in the Raman spectrum. The distance-dependent Raman spectra in Fig. 6 show significant blue-shifting only for $\mathrm{OH}$ groups with the hydrogen within $2 \AA$ of a pore oxygen atom, i.e., donating an H-bond or close enough to the surface to do so. The higher surface silanol density in pore 2 in conjunction with the slightly weaker blue-shifted peak intensity suggests that the surface silanols reduce the number of waters donating $\mathrm{H}$-bonds to the pore surface. The silanols presumably instead themselves donate $\mathrm{H}$-bonds to interfacial water molecules. It is possible that the neglect of the direct contributions to the spectra from the silanols themselves (Sec. II A), would partially negate these small differences between the pores. The overall result, however, is that comparison of the results from the two pores indicates that the spectra depend quite weakly on the surface $\mathrm{OH}$ density and the atomistic details of the pore structure, ${ }^{32}$ even though it is the spectra of the waters next to the pore interface that are most strongly modified relative to the bulk liquid. This is somewhat surprising and certainly not positive news for interrogating water-filled mesoporous material structure with vibrational spectroscopic techniques; we note, however, that other liquids, e.g., acetonitrile, have vibrational spectra that are quite sensitive to the silanol density. ${ }^{32}$

\section{CONCLUDING REMARKS}

The empirical frequency mapping approach has been applied straightforwardly to water in silica pores. The results show that this method can reproduce the basic features of the measured Raman and linear IR spectra of water in 
mesoporous silica. Interestingly, the results are not sensitive to the precise method for calculating the electric field from the silica. However, in the future, improved modeling may be required to describe the spectra with greater quantitative accuracy once more direct comparisons are available (i.e., for identical pore sizes and isotopic dilution).

The key effect observed for water in the nanoscale silica pores is blueshifting of the $\mathrm{OH}$ frequency, relative to bulk liquid water, for those groups donating $\mathrm{H}$-bonds to the pore surface. These weaker $\mathrm{H}$-bonds lead to $\mathrm{OH}$ frequencies that are $\sim 100 \mathrm{~cm}^{-1}$ higher than both that in bulk water and those OHs in the pore that donate $\mathrm{H}$-bonds to other waters. These results indicate that the effect of "confinement" on the spectra is, in fact, limited to these distinctly interfacial water molecules and would be expected to be similar for planar water/silica interfaces.

The linear IR spectrum of $\mathrm{HOD} / \mathrm{D}_{2} \mathrm{O}$ shows little effect upon confinement in these $\sim 2.4 \mathrm{~nm}$ diameter silica pores. This is a consequence of the weaker H-bonds, which in addition to having higher vibrational frequencies, exhibit reduced transition dipole moments that decrease their contributions to the IR spectrum. These non-Condon effects mean that the IR spectrum of confined water contains virtually no information about the interfacial water molecules donating $\mathrm{H}$-bonds to the pore surface. Thus, linear IR spectroscopy is not the best probe of water confined in mesoporous silica.

In contrast, the Raman spectrum has significantly weaker non-Condon effects because the transition polarizability is less sensitive to the environment and frequency of the $\mathrm{OH}$. The Raman spectrum of confined water therefore exhibits a significant blue-shifted shoulder that is not present in bulk water and reports on the interfacial waters engaged in weaker $\mathrm{H}$-bonds with the surface. These results indicate that Raman spectroscopy can provide greater insight into silica-confined water.

Predictions of the 2D-IR photon echo spectra, which has not yet been measured, are presented. While the spectra show the same non-Condon effects that reduce the importance of the interfacial waters in the linear IR spectrum, the slower spectral diffusion is apparent. This should be measurable in an experiment through the center-line slope analysis, ${ }^{81}$ which shows the retarded dynamics in the present simulations. In addition, it is shown that the center-line slope reasonably represents the calculated frequency autocorrelation function, supporting its interpretation.

The results presented here provide guidance on the strengths and limitations of vibrational spectroscopy, by elucidating what features of the strongly modified structure and dynamics of nanoconfined liquids are (and are not) manifested in various spectra. At the same time, they point to the need for further progress in experimental probes of confined liquids along with continued connections between theory and measurements to aid interpretation. In the near term, study of other liquids and solutions should provide some additional fundamental insight through examination of different frequency shift, non-Condon, and resonant vibrational coupling effects as well as access to longer dynamical timescales due to longer vibrational relaxation times.

\section{ACKNOWLEDGMENTS}

W.H.T. acknowledges support for this work from the Chemical Sciences, Geosciences, and Biosciences Division, Office of Basic Energy Sciences, Office of Science, U.S. Department of Energy (Grant No. DE-FG02-05ER15708). D.L. acknowledges support from the Agence Nationale de la Recherche (Grant No. ANR-2011-BS08-010-01).

${ }^{1}$ J. C. Rasaiah, S. Garde, and G. Hummer, Annu. Rev. Phys. Chem. 59, 713 (2008).

${ }^{2}$ N. E. Levinger and L. A. Swafford, Annu. Rev. Phys. Chem. 60, 385 (2009).

${ }^{3}$ M. A. Ricci, F. Bruni, and A. Giuliani, Faraday Discuss. 141, 347 (2009).

${ }^{4}$ M. D. Fayer, D. E. Moilanen, D. B. Wong, D. E. Rosenfeld, E. E. Fenn, and S. Park, Acc. Chem. Res. 42, 1210 (2009).

${ }^{5}$ M. D. Fayer and N. E. Levinger, Annu. Rev. Anal. Chem. 3, 89 (2010).

${ }^{6}$ R. Richert, Annu. Rev. Phys. Chem. 62, 65 (2011).

${ }^{7}$ W. H. Thompson, Annu. Rev. Phys. Chem. 62, 599 (2011).

${ }^{8}$ N. Giovambattista, P. J. Rossky, and P. G. Debenedetti, Annu. Rev. Phys. Chem. 63, 179 (2012).

${ }^{9}$ J. Schroeder, V. H. Schiemann, P. T. Sharko, and J. Jonas, J. Chem. Phys. 66, 3215 (1977).

${ }^{10}$ L. Nikiel, B. Hopkins, and T. W. Zerda, J. Phys. Chem. 94, 7458 (1990).

${ }^{11}$ S. L. Wallen, L. Nikiel, J. Yi, and J. Jonas, J. Phys. Chem. 99, 15421 (1995).

${ }^{12}$ J. Yi and J. Jonas, J. Phys. Chem. 100, 16789 (1996).

${ }^{13}$ C. Czeslik, Y. J. Kim, and J. Jonas, J. Raman Spectrosc. 31, 571 (2000).

${ }^{14}$ R. S. Luo and J. Jonas, J. Raman Spectrosc. 32, 975 (2001).

${ }^{15}$ S. Kittaka, T. Iwashita, A. Serizawa, M. Kranishi, S. Takahara, Y. Kuroda, T. Mori, and T. Yamaguchi, J. Phys. Chem. B 109, 23162 (2005).

${ }^{16}$ T. Yamaguchi, K. Yoshida, P. Smirnov, T. Takamuku, S. Kittaka, S. Takahara, Y. Kuroda, and M.-C. Bellissent-Funel, Eur. Phys. J.-Spec. Top. 141, 19 (2007).

${ }^{17}$ I. R. Piletic, D. E. Moilanen, D. B. Spry, N. E. Levinger, and M. D. Fayer, J. Phys. Chem. A 110, 4985 (2006).

${ }^{18}$ D. E. Moilanen, N. E. Levinger, D. B. Spry, and M. D. Fayer, J. Am. Chem. Soc. 129, 14311 (2007)

${ }^{19}$ S. Park, D. E. Moilanen, and M. D. Fayer, J. Phys. Chem. B 112, 5279 (2008).

${ }^{20}$ D. E. Moilanen, E. E. Fenn, D. B. Wong, and M. D. Fayer, J. Phys. Chem. B 113, 8560 (2009).

${ }^{21}$ D. E. Moilanen, E. E. Fenn, D. B. Wong, and M. D. Fayer, J. Am. Chem. Soc. 131, 8318 (2009).

${ }^{22}$ D. E. Moilanen, E. E. Fenn, D. B. Wong, and M. D. Fayer, J. Chem. Phys. 131, 014704 (2009).

${ }^{23}$ R. Musat, J. P. Renault, M. Candelaresi, D. J. Palmer, S. Le Caër, R. Righini, and S. Pommeret, Angew. Chem., Int. Ed. 47, 8033 (2008).

${ }^{24}$ X. F. Huang, Q. Wang, X. X. Liu, S. H. Yang, C. X. Li, G. Sun, L. Q. Pan, and K. Q. Lu, J. Phys. Chem. C 113, 18768 (2009).

${ }^{25}$ S. Le Caër, S. Pin, S. Esnouf, Q. Raffy, J. P. Renault, J. B. Brubach, G. Creff, and P. Roy, Phys. Chem. Chem. Phys. 13, 17658 (2011).

${ }^{26}$ S. Le Caër, M. Lima, D. Gosset, D. Simeone, F. Bergaya, S. Pommeret, J. P. Renault, and R. Righini, J. Phys. Chem. C 116, 12916 (2012).

${ }^{27}$ R. Costard, N. E. Levinger, E. T. J. Nibbering, and T. Elsaesser, J. Phys. Chem. B 116, 5752 (2012).

${ }^{28}$ R. Costard and T. Elsaesser, J. Phys. Chem. B 117, 15338 (2013).

${ }^{29}$ A. A. Bakulin, D. Cringus, P. A. Pieniazek, J. L. Skinner, T. L. C. Jansen, and M. S. Pshenichnikov, J. Phys. Chem. B 117, 15545 (2013).

${ }^{30}$ P. A. Pieniazek, Y.-S. Lin, J. Chowdhary, B. M. Ladanyi, and J. L. Skinner, J. Phys. Chem. B 113, 15017 (2009).

${ }^{31}$ T. S. Gulmen and W. H. Thompson, Langmuir 22, 10919 (2006).

${ }^{32}$ C. M. Morales and W. H. Thompson, J. Phys. Chem. A 113, 1922 (2009).

${ }^{33}$ J. Faeder and B. M. Ladanyi, J. Phys. Chem. B 104, 1033 (2000).

${ }^{34}$ J. Chowdhary and B. M. Ladanyi, J. Phys. Chem. B 113, 15029 (2009).

${ }^{35}$ T. Takamuku, H. Maruyama, S. Kittaka, S. Takahara, and T. Yamaguchi, J. Phys. Chem. B 109, 892 (2005).

${ }^{36}$ T. Yamaguchi, H. Sugino, K. Ito, K. Yoshida, and S. Kittaka, J. Mol. Liq. 164, 53 (2011).

${ }^{37}$ J. A. Harvey and W. H. Thompson, J. Phys. Chem. B 119, 9150 (2015).

${ }^{38}$ J. Rodriguez, M. Dolores Elola, and D. Laria, J. Phys. Chem. B 114, 7900 (2010).

${ }^{39}$ A. A. Milischuk and B. M. Ladanyi, J. Chem. Phys. 135, 174709 (2011).

${ }^{40}$ G. Liu, Y.-Z. Li, and J. Jonas, J. Chem. Phys. 95, 6892 (1991).

${ }^{41}$ N. Koone, Y. Shao, and T. W. Zerda, J. Phys. Chem. 99, 16976 (1995). 
${ }^{42}$ A. Scodinu and J. T. Fourkas, J. Phys. Chem. B 106, 10292 (2002).

${ }^{43}$ R. A. Farrer and J. T. Fourkas, Acc. Chem. Res. 36, 605 (2003).

${ }^{44}$ S. Takahara, N. Sumiyama, S. Kittaka, T. Yamaguchi, and M.-C. BellissentFunel, J. Phys. Chem. B 109, 11231 (2005).

${ }^{45}$ S. Takahara, S. Kittaka, T. Mori, Y. Kuroda, T. Takamuku, and T. Yamaguchi, J. Phys. Chem. C 112, 14385 (2008).

${ }^{46}$ D. Laage and W. H. Thompson, J. Chem. Phys. 136, 044513 (2012).

${ }^{47}$ A. C. Fogarty, E. Duboué-Dijon, D. Laage, and W. H. Thompson, J. Chem. Phys. 141, 18C523 (2014).

${ }^{48}$ A. Taschin, P. Bartolini, A. Marcelli, R. Righini, and R. Torre, Faraday Discuss. 167, 293 (2014).

${ }^{49}$ A. Taschin, P. Bartolini, A. Marcelli, R. Righini, and R. Torre, J. Phys.: Condens. Matter 27, 194107 (2015).

${ }^{50} \mathrm{P}$. Gallo, M. Rovere, M. A. Ricci, C. Hartnig, and E. Spohr, Europhys. Lett. 49, 183 (2000).

${ }^{51}$ P. Gallo, M. Rovere, and S.-H. Chen, J. Phys.: Condens. Matter 22, 284102 (2010).

${ }^{52}$ K. Shirono and H. Daiguji, J. Phys. Chem. C 111, 7938 (2007).

${ }^{53}$ Q. Zhang, K.-Y. Chan, and N. Quirke, Mol. Simul. 35, 1215 (2009).

${ }^{54}$ A. A. Milischuk, V. Krewald, and B. M. Ladanyi, J. Chem. Phys. 136, 224704 (2012).

${ }^{55}$ A. A. Milischuk and B. M. Ladanyi, J. Chem. Phys. 141, 18C513 (2014).

${ }^{56}$ S. J. Plimpton, J. Comput. Phys. 117, 1 (1995).

${ }^{57} \mathrm{See}$ http://lammps.sandia.gov for The LAMMPS molecular dynamics package.

${ }^{58}$ See http://towhee.sourceforge.net for The Towhee Monte Carlo simulation code.

${ }^{59}$ H. J. C. Berendsen, J. R. Grigera, and T. P. Straatsma, J. Phys. Chem. 91, 6269 (1987).

${ }^{60}$ T. S. Gulmen and W. H. Thompson, in Dynamics in Small Confining Systems VIII, edited by J. T. Fourkas, P. Levitz, R. Overney, and M. Urbakh (Materials Research Society, Warrendale, PA, 2005).

${ }^{61}$ S. Nose, Mol. Phys. 52, 255 (1984).

${ }^{62}$ W. G. Hoover, Phys. Rev. A 31, 1695 (1985).
${ }^{63}$ S. A. Corcelli, C. P. Lawrence, and J. L. Skinner, J. Chem. Phys. 120, 8107 (2004).

${ }^{64}$ B. Auer, R. Kumar, J. R. Schmidt, and J. L. Skinner, Proc. Natl. Acad. Sci. U. S. A. 104, 14215 (2007).

${ }^{65}$ S. M. Gruenbaum, C. J. Tainter, L. Shi, Y. Ni, and J. L. Skinner, J. Chem. Theor. Comput. 9, 3109 (2013).

${ }^{66}$ Y.-S. Lin, B. M. Auer, and J. L. Skinner, J. Chem. Phys. 131, 144511 (2009).

${ }^{67}$ S. M. Gruenbaum, P. A. Pieniazek, and J. L. Skinner, J. Chem. Phys. 135, 164506 (2011)

${ }^{68}$ S. Roy, S. M. Gruenbaum, and J. L. Skinner, J. Chem. Phys. 141, 18 C502 (2014).

${ }^{69}$ See supplementary material at http://dx.doi.org/10.1063/1.4949766 for figures showing the dependence of the 2D-IR photon echo spectra on the method used to calculate the electric field and comparisons of the linear IR spectrum, 2D-IR spectra, and frequency autocorrelation functions for the two pores simulated.

${ }^{70}$ C. J. Fecko, J. J. Loparo, S. T. Roberts, and A. Tokmakoff, J. Chem. Phys. 122, 054506 (2005).

${ }^{71}$ G. Onori and A. Santucci, J. Phys. Chem. 97, 5430 (1993).

${ }^{72}$ N. V. Nucci and J. M. Vanderkooi, J. Phys. Chem. B 109, 18301 (2005).

${ }^{73}$ I. R. Piletic, H.-S. Tan, and M. D. Fayer, J. Phys. Chem. B 109, 21273 (2005).

${ }^{74}$ S. A. Corcelli and J. L. Skinner, J. Phys. Chem. A 109, 6154 (2005).

${ }^{75}$ V. Crupi, F. Longo, D. Majolino, and V. Venuti, Eur. Phys. J.-Spec. Top. 141, 61 (2007).

${ }^{76}$ V. Crupi, S. Interdonato, F. Longo, D. Majolino, P. Migliardo, and V. Venuti, J. Raman Spectrosc. 39, 244 (2008).

${ }^{77}$ D. E. Hare and C. M. Sorensen, J. Chem. Phys. 96, 13 (1992).

${ }^{78}$ B. M. Auer and J. L. Skinner, J. Chem. Phys. 128, 224511 (2008).

${ }^{79}$ J. R. Schmidt, S. T. Roberts, J. J. Loparo, A. Tokmakoff, M. D. Fayer, and J. L. Skinner, Chem. Phys. 341, 143 (2007).

${ }^{80}$ P. Hamm and M. Zanni, Concepts and Methods of $2 D$ Infrared Spectroscopy (Cambridge University Press, New York, 2011).

${ }^{81}$ K. Kwak, D. E. Rosenfeld, and M. D. Fayer, J. Chem. Phys. 128, 204505 (2008). 